\title{
Complicaciones durante la neuromodulación de raíces sacras en el tratamiento de la incontinencia fecal grave*
}

\author{
Drs. PEDRO MOYA F. ${ }^{1}$, ANTONIO ARROYO S. ${ }^{1}$, JAVIER LACUEVA G. ${ }^{1}$, \\ FERNANDO CANDELA P. ${ }^{1}$, RAFAEL CALPENA R. ${ }^{1}$ \\ 1 Departamento de Cirugía, Hospital General Universitario de Elche, Alicante. \\ España.
}

\begin{abstract}
Complications during sacral nerve stimulation in the treatment of severe fecal incontinence

Objective: To evaluate the complications of sacral nerve stimulation for the treatment of fecal incontinence. Background: Sacral nerve stimulation is considered to be nowadays an effective treatment for fecal incontinence in selected patients. Many authors have reported excellent results and indication for his use have increased. Nevertheless, even being a simple technique, not it exempts this from complications. Methods: Fifty-two patients with severe faecal incontinence, treated with sacral nerve stimulation between January 2002 and December 2010, were analysed. All adverse events during follow-up were recorded. Results: There was not surgical complication during peripheral neural evaluation test. In our series, with an average follow-up to $55.52 \pm 31.84$ months (range: 12-121), we had a rate of adverse effects of $14 \%$, infection of surgical wound in two patients, break of electrode in two patients and the presence of pain in the place of the implant, with episodes of extremity pain and paresthesias in low members in 3 patients. Conclusions: Sacral nerve stimulation in severe fecal incontinence is a safe treatment, with a low index of complications.
\end{abstract}

Key words: Sacral nerve stimulation, fecal incontinence, complications, adverse effects.

\section{Resumen}

Objetivo: Evaluar las complicaciones de la neuromodulación de raíces sacras en el tratamiento de la incontinencia fecal. Introducción: La neuromodulación de raíces sacras ha sido descrita como un tratamiento quirúrgico eficaz en pacientes con incontinencia fecal grave. Muchos autores han comunicado excelentes resultados y las indicaciones en su uso han aumentado. Sin embargo, a pesar de ser una técnica quirúrgica sencilla, no está exenta de complicaciones. Material y Método: 52 pacientes con incontinencia fecal grave, tratados mediante neuromodulación de raíces sacras entre enero de 2002 y diciembre de 2010, fueron anali-

*Recibido el 8 de abril de 2013 y aceptado para publicación el 8 de mayo de 2013.

No existen conflictos de interés.

Correspondencia: Dr. Pedro Moya F.

Camino de los Garres 21. 30012 Murcia, España.

pedromoyaforcen@gmail.com 
zados. Todas las complicaciones, tanto durante la implantación del dispositivo como durante el seguimiento, fueron registradas. Resultados: No se registraron complicaciones durante la prueba de estimulación. En nuestra serie, con un seguimiento de 55,52 $\pm 31,84$ meses, observamos un 14\% de efectos adversos, infección de la herida quirúrgica en dos pacientes, rotura o movilización del electrodo en 2 pacientes y dolor y parestesias en 3 pacientes. Discusión: La neuroestimulación de raíces sacras en el tratamiento de la incontinencia fecal es un tratamiento seguro, con un bajo índice de complicaciones.

Palabras clave: Neuromodulación de raíces sacras, incontinencia fecal, complicaciones, efectos adversos.

\section{Introducción}

La incontinencia fecal (IF) representa un complejo y multifactorial problema de la salud ${ }^{1}$, que provoca situaciones embarazosas que pocas personas pueden tolerar. La IF es definida como la pérdida parcial o total de la capacidad para controlar voluntariamente la expulsión de heces y gases. La gravedad de la IF viene reflejada por la frecuencia y el tipo de pérdidas que presentan los pacientes ${ }^{2,3}$.

La neuromodulación de raíces sacras (NRS) es considerada, hoy día, un tratamiento eficaz para la incontinencia fecal en pacientes seleccionados. Desde que en 1995, cuando Matzel et $\mathrm{al}^{4}$, comunicaron el empleo de la NRS para el tratamiento de la incontinencia fecal, muchos autores han relatado resultados excelentes y la indicación para su empleo ha aumentado. Sin embargo, aún siendo una técnica sencilla, no está exenta de complicaciones.

El objetivo de este estudio es evaluar las complicaciones de la NRS en el tratamiento de incontinencia fecal.

\section{Material y Método}

Estudio prospectivo, observacional y longitudinal, de una serie de casos llevado a cabo entre enero de 2002 y diciembre de 2010 , en todos los pacientes diagnosticados de incontinencia fecal grave en el Hospital General Universitario de Elche y subsidiarios de implante de un neuromodulador de raíces sacras.

Para ser incluidos en el estudio, los pacientes debieron recibir tratamiento conservador, en el que se incluía tratamiento farmacológico, dieta astringente y fisioterapia del suelo pélvico, sin resultados, antes de la cirugía. El resultado obtenido en la escala de Wexner debía ser superior a 12 puntos.

Embarazadas, pacientes menores de 18 años, la presencia de infección local aguda o crónica, coagulopatía, marcapasos o arritmias cardíacas, enfermedad oncológica con expectativa de vida inferior a 5 años, colostomía, y pacientes con alteraciones psiquiátricas que hicieran imposible el correcto seguimiento del protocolo, fueron excluidos.
Las pruebas preoperatorias incluyeron examen físico completo, manometría endoanal y ecografía rectoanal. Los datos fueron recogidos según un protocolo de neuroestimulación de raíces sacras creado específicamente para este estudio. La continencia anal fue evaluada usando la escala de Wexner ${ }^{5}$ y el resultado fue obtenido a partir de un diario defecatorio que completaron los pacientes durante 21 días.

\section{Técnica quirúrgica}

La técnica quirúrgica para la implantación del neuroestimulador de raíces sacras ha sido descrita anteriormente en detalle ${ }^{6}$. Un electrodo tetrapolar (Medtronic Modelo 3889) es introducido bajo anestesia local en el foramen S3 o S4 basado en la mejor respuesta sensitiva o motora durante la fase de evaluación periférica, y es conectado percutáneamente mediante un kit de extensión (Medtronic Modelo 9550-05) a un generador externo (Medtronic Modelo 3625). El correcto posicionamiento de electrodo durante el procedimiento se realiza mediante radiología. Durante los 21 días siguientes a la intervención, el paciente debe cumplimentar un diario defecatorio, donde se recogen detalladamente los episodios de urgencia evacuatoria e incontinencia fecal. Si el paciente ha presentado una mejoría superior al 50\% de los episodios de incontinencia fecal respecto a su situación basal o una reducción del 50\% de los días con incontinencia fecal, se realiza la implantación permanente del neuroestimulador de raíces sacras. El electrodo tetrapolar es conectado a un generador (Medtronic Modelo 3023 InterStim I) mediante un kit de extensión (Medtronic Modelo 3095) o directamente al generador (Medtronic Modelo 3058 InterStim II) y es situado subcutáneamente en la zona glútea.

\section{Profilaxis}

Todos los pacientes recibieron profilaxis antibiótica 30 min antes de la prueba de estimulación y antes de la implantación del generador definitivo. El antibiótico usado fue la Piperacilina-Tazobactan, $4 / 0,5 \mathrm{~g}$, de acuerdo con la recomendación del grupo español de neuromodulación en disfunción anal (GENDA). Durante la cirugía, cada uno de los implantes fueron sumergidos en una solución de gen- 
tamicina ( $80 \mathrm{mg}$ de gentamicina en $500 \mathrm{ml}$ de suero fisiológico) antes de su implantación. Así mismo, los pacientes recibieron profilaxis antitrombótica mediante enoxaparina 40 mg subcutánea.

\section{Seguimiento}

Tras la implantación definitiva del estimulador de raíces sacras, a los pacientes se le realizaban controles en consultas externas de Coloproctología, al primer mes, al sexto mes y anualmente. Todos los efectos adversos y complicaciones fueron registrados.

\section{Resultados}

\section{Pacientes y características poblacionales}

Un total de 52 pacientes, constituido por 10 varones y 42 mujeres con una mediana de edad de 63,5 años (rango: 22-77), en los que se les realizó neuromodulación de raíces sacras durante el período de enero de 2002 a diciembre de 2010, y que cumplían los criterios de inclusión anteriormente descritos, fueron incluidos en el presente estudio. Las características de estos pacientes se describen en la Tabla 1.

\section{Implante quirúrgico}

En 52 pacientes se realizó la prueba de estimulación. En 27 pacientes la raíz con mayor respuesta sensitiva y motora fue S3 (13 pacientes (25\%) S3 derecha y 14 pacientes (26,9\%) S3 izquierda). En 25 pacientes la raíz estimulada fue S4 (14 pacientes $(26,9 \%) \mathrm{S} 4$ derecha y 11 pacientes $(21,2 \%) \mathrm{S} 4$ izquierda).

En 50 de los 52 pacientes $(96,15 \%)$, se produjo una reducción igual o superior al $50 \%$ de los episodios o de los días con episodios de incontinencia fecal, por lo que se le calificó de aptos para la implantación del generador definitivo.

\section{Seguimiento}

Ninguno de los pacientes fue perdido durante el seguimiento. El seguimiento medio de los pacientes incluidos en el presente estudio fue de 55,52 \pm 31,841 meses, con un rango de 12-121 meses.

\section{Efecto en la incontinencia fecal}

La mediana de la puntuación obtenida en el Wexner preoperatorio decreció con diferencias estadísticamente significativas desde un valor de 15 rango 13-20 (preoperatorio) a 4 rango 0-7 (revisión a los 6 meses). Estas diferencias se mantienen constantes a lo largo del seguimiento.

\section{Complicaciones}

No se objetivaron complicaciones quirúrgicas durante la implantación del electrodo o el período de estimulación diagnóstica. De los 50 sujetos a los que se les implantó el generador definitivo, dos de ellos (4\%) experimentaron infección de la herida quirúrgica, con posterior contaminación del generador. Se intentó tratamiento conservador con antibióticos intravenosos y cura local, con mala evolución, por lo que se decidió retirada del dispositivo y nuevo implante a los dos meses, sin complicaciones posteriores y obteniendo los mismos resultados funcionales que durante la primera implantación.

Tres pacientes (6\%) experimentaron a lo largo del seguimiento molestias en el lugar de implantación, acompañado de dolor extremadamente agudo en extremidades inferiores y parestesias. Esta clínica fue refractaria a todo tratamiento médico que se intentó, por lo que se decidió el explante de los neuroestimuladores. En otro paciente, tras caída, el neuroestimulador dejó de funcionar. Se comprobó que el electrodo se había roto, sin desplazamiento del mismo, por lo que se procedió a su sustitución de éste con anestesia local. En otra paciente, el elec-

Tabla 1. Pacientes incluidos

\begin{tabular}{|lc|}
\hline Edad & 63,5 (Rango: 22-77) \\
Sexo (M/V) & $42 / 10$ \\
Duración de los síntomas (años) & 2 - 36 \\
Embarazos & 2 (Rango: 0-8) \\
Cirugía previa & \\
Esfinteroplastía & 5 \\
Reparación post-anal & 4 \\
Resección anterior & 4 \\
Hemorroidectomía & 4 \\
Fistulotomía y esfinteroplastía & 10 \\
Esfinterotomía lateral interna & 3 \\
Colgajo de Fergunson & 1 \\
Histerectomía & 12 \\
Prolapso uterino & 1 \\
Prolapso rectal & 2 \\
Prostatectomía & 2 \\
Etiología & 13 \\
Cirugía anal & 22 \\
Lesiones obstétricas & 4 \\
Postmenopáusica & 2 \\
Resección anterior baja & 1 \\
Radioterapia & 2 \\
Congénita & \\
Paraplejia & \\
Polineuropatía viral & \\
Esclerodermia & \\
Incontinencia urinaria & \\
\hline
\end{tabular}


trodo se movilizó durante un parto vaginal por lo que hubo que reemplazarlo mediante intervención con anestesia local.

\section{Reemplazo del generador}

En el momento del cierre del seguimiento para redactar el presente trabajo, en 3 pacientes se había realizado el cambio del neuroestimulador definitivo por agotamiento de la batería. En el primer paciente ocurrió tras 62 meses, en el segundo a los 83 meses y en el tercero a los 100 meses tras su implantación. Finalmente, en otro paciente, se realizó explante del neuroestimulador al ser necesario realizar una resonancia magnética nuclear.

\section{Discusión}

Cuando las medidas médicas se han agotado, el tratamiento quirúrgico es la única opción para pacientes con incontinencia fecal. Las opciones quirúrgicas incluían la reparación post-anal, esfinteroplastía anterior, transposiciones musculares y el esfínter anal artificial. Desafortunadamente, los resultados de estos procedimientos no presentan siempre buenos resultados, con bastantes efectos adversos o resultados pobres a largo plazo ${ }^{7-14}$.

El mayor problema que se presenta, a la hora de estudiar las complicaciones durante las distintas fases de la neuromodulación de raíces sacras, es la poca homogeneidad presente en la literatura a la hora de reportar las complicaciones y efectos adversos aparecidos durante el tratamiento. Mientras que algunos autores únicamente consideran como efectos adversos a las complicaciones propias de la cirugía (infección, serosa, hematoma...) o a los fallos o roturas en el material implantable o utilizado para las distintas pruebas, otros comunican cualquier tipo de alteración (dolor, parestesias, cambios en la sensación...) como efectos adversos.

\section{Fase de evaluación periférica}

Durante la fase de evaluación periférica (PNE), en la que se seleccionan aquellos pacientes que más se van a beneficiar de la terapia neuromoduladora, es frecuente la comunicación de complicaciones como infección del sitio quirúrgico, erosiones en la piel y dolor y parestesias en miembros inferiores. La complicación más comúnmente descrita es el desplazamiento del electrodo, con una frecuencia cercana al 6\%, que ocurría principalmente cuando se usaba el electrodo "tined lead"15. Actualmente, con el uso del electrodo tetrapolar, los desplazamientos del electrodo durante la fase de evaluación periférica son más difíciles. Fases de evaluación prolongadas (más de 21 días) podrían facilitar el desplazamiento del electrodo. En nuestra serie, ninguno de los pacientes experimentó este tipo de complicación.

Wexner et $\mathrm{al}^{16}$, en un estudio multicéntrico llevado a cabo en 16 centros de Norte América y Australia, sobre 129 pacientes en los que se realizó la prueba de estimulación subcrónica, reportan un $12 \%$ de efectos adversos, entre los que se encontraban irritación de la piel en la zona de localización del electrodo, migración y rotura del electrodo, hematomas y como más frecuente, dolor durante la estimulación. Rasmussen et $\mathrm{al}^{17}$, comunican una tasa de complicaciones durante el test de estimulación cercana al $20 \%$, con un $6 \%$ de infecciones y cerca de un $14 \%$ de problemas relacionados con el electrodo.

Durante esta fase, no experimentamos ninguna complicación infecciosa. El uso de profilaxis antibiótica sistémica, así como el sumergir todo el material implantable en una solución antibiótica, es la medida más importante para minimizar las complicaciones infecciosas durante esta fase.

\section{Fase de implantación del generador definitivo}

Tras la implantación del generador definitivo, Jarrett et $a^{18}$, en una revisión sistemática (149 pacientes) comunican una frecuencia de efectos adversos cercana al $13 \%$. Chan et $\mathrm{al}^{19}$, con un seguimiento de 12 meses, presentaron un $15 \%$ de complicaciones menores. Se ha visto que a mayor seguimiento, mayores son las complicaciones descritas. Melenhorst et $\mathrm{al}^{20}$, con un seguimiento medio de 25 meses comunican unas complicaciones de 19\%, Michelsen et $\mathrm{al}^{21}$, un $25 \%$ con el mismo seguimiento. Faucheron et $\mathrm{al}^{22}$, con un seguimiento medio de 48,5 meses, describen complicaciones en el 41,3\% de los pacientes, que requirieron revisión quirúrgica del sistema, con un $25 \%$ de explantes. Matzel et $\mathrm{al}^{23}$, en la serie, con mayor seguimiento, cercano a 10 años, pero con un número muy limitado de pacientes (12 pacientes), reportan un $41,6 \%$ de complicaciones.

En nuestra serie, con un seguimiento medio superior a 55 meses, hemos tenido una tasa de efectos adversos del $12 \%$, infección de herida quirúrgica en dos pacientes, rotura de electrodo tras caída en un pacientes y la presencia de dolor en el lugar del implante, con episodios de dolor agudo y parestesias en miembros inferiores en 3 pacientes, complicaciones de las más bajas comunicadas.

La indicación más frecuente de explante del dispositivo es el fallo inexplicable de la terapia a lo largo del seguimiento (Tabla 2). Faucheron et $\mathrm{a}^{22}$, comunican la retirada del $10 \%$ de los dispositivos implantados. Mismos resultados presentados por Michelsen et $\mathrm{al}^{21}$. Rasmussen et $\mathrm{al}^{17}$, comunican el explante de 3 de los 37 dispositivos que implantaron por cese de respuesta clínica tras 3, 12 y 18 meses. Melenhorst et $\mathrm{al}^{20}$, describen un fallo del dispositivo, 
Tabla 2. Fallos reportados tras la implantación del generador definitivo

\begin{tabular}{|c|c|c|c|c|}
\hline & $\begin{array}{c}\text { Prueba } \\
\text { estimulación }\end{array}$ & $\begin{array}{c}\text { Pacientes } \\
\text { implantados }\end{array}$ & $\begin{array}{c}\text { Seguimiento medio } \\
\text { (meses) }\end{array}$ & $\begin{array}{c}\text { Pérdida de eficacia } \\
(\%)\end{array}$ \\
\hline Presente estudio & 52 & 50 & 55 & 0 \\
\hline Altamore et $\mathrm{al}^{37}$ & 41 & 60 & 14 & 22 \\
\hline Boyle et al ${ }^{38}$ & 15 & 13 & - & 23 \\
\hline Faucheron et $\mathrm{al}^{22}$ & 40 & 29 & - & 3 \\
\hline Gourcerol et al ${ }^{39}$ & 61 & 33 & 12 & 27 \\
\hline Govaert et $\mathrm{al}^{40}$ & ND & 155 & 28,1 & 6 \\
\hline Hetzer et $\mathrm{al}^{41}$ & 44 & 37 & - & 5 \\
\hline Holzer et $\mathrm{al}^{42}$ & 7 & 7 & 32 & 14 \\
\hline Jarret et $\mathrm{al}^{43}$ & 59 & 46 & 12 & 4 \\
\hline Leroi et $\mathrm{al}^{44}$ & 9 & 6 & 2 & 17 \\
\hline Malouf et $\mathrm{al}^{45}$ & 5 & 5 & 16 & 20 \\
\hline Matzel et a ${ }^{46}$ & 37 & 34 & 24 & 9 \\
\hline Matzel et $\mathrm{al}^{23}$ & NR & 12 & 9,8 años & 8 \\
\hline Melenhorst et $\mathrm{al}^{26}$ & 134 & 100 & 25,5 & 21 \\
\hline Michelsen et al ${ }^{27}$ & 177 & 142 & 24 & 20 \\
\hline Muñoz-Duyos et $\mathrm{al}^{47}$ & 47 & 29 & 34,7 & 28 \\
\hline Rasmussen et a $1^{17}$ & 43 & 37 & 6 & 8 \\
\hline Ratto et $\mathrm{al}^{48}$ & 10 & 10 & 33 & 0 \\
\hline Tjandra et $\mathrm{al}^{25}$ & 60 & 53 & 12 & 0 \\
\hline Uludag et $\mathrm{al}^{49}$ & 75 & 62 & 12 & 3 \\
\hline Wexner et $\mathrm{al}^{16}$ & 133 & 120 & 28 & 5 \\
\hline
\end{tabular}

que previamente funcionaba, en el $20 \%$ de sus pacientes con una media de 13,6 meses tras su implantación. Un nuevo test de estimulación sobre estos pacientes, produjo buena respuesta en el $25 \%$ de estos pacientes. Una fibrosis alrededor de los electrodos, causada por la corriente eléctrica, explicaría este tipo de fallos y la buena respuesta de algunos de estos pacientes a un nuevo test de estimulación, sobre una raíz contralateral. En nuestra serie, ningún paciente experimentó este fallo inexplicable de la terapia, a pesar de un seguimiento medio superior a 55 meses.

La infección del implante es otra causa frecuente de explante (Tabla 3). La tasa de infección es descrita entre el $1 \%$ y el $17 \%$. Wexner et $\mathrm{al}^{16}$, en el estudio multicéntrico, llevado a cabo en Norte América y Australia, describen un 10,8\% de infecciones. Este alto índice, probablemente, refleja en parte, la curva de aprendizaje. Rasmussen et $\mathrm{al}^{17}$, reportan una tasa de infección del $5 \%$. En el multicéntrico francés ${ }^{24}$, la tasa descrita, es muy inferior, $1,5 \%$, misma cifra comunicada por Michelsen et $\mathrm{al}^{21}$. Tjandra et $\mathrm{a}^{25}$, no presentaron ninguna infección, sobre 60 pacientes. En la mayoría de las series, cuando la infección es leve, un tratamiento antibiótico y curas locales suele ser suficiente. En caso de infecciones más importantes, el explante, el tratamiento antibiótico y las curas locales, y un implante posterior suele dar resultados clínicos similares a los obtenidos antes de presentar la infección. En nuestro trabajo, el índice de infecciones fue de 4\% (2 pacientes). Esta complicación, aunque molesta, se pudo resolver con el explante del dispositivo y reimplante dos meses después tras tratamiento antibiótico y curas locales. De cualquier modo las complicaciones obtenidas son muy inferiores a las que se alcanzan con otros tratamientos quirúrgicos.

El dolor en la zona del implante es la complicación más frecuente de la neuroestimulación y otra causa de explante, si bien en la mayoría de los casos un tratamiento médico es suficiente para controlar el cuadro y no es necesaria la retirada del generador. 
Tabla 3. Complicaciones reportadas tras la implantación del generador definitivo

\begin{tabular}{|c|c|c|c|c|c|c|c|}
\hline & $\begin{array}{c}\text { Pacientes } \\
\text { implantados }\end{array}$ & $\begin{array}{l}\text { Seguimiento } \\
\text { medio (meses) }\end{array}$ & Dolor & Infección & $\begin{array}{l}\text { Rotura } \\
\text { electrodo }\end{array}$ & Otros & $\begin{array}{c}\text { Revisiones } \\
\text { quirúrgicas }\end{array}$ \\
\hline Presente estudio & 50 & 55 & 3 & 2 & 2 & - & 4 \\
\hline Altamore et $\mathrm{al}^{37}$ & 60 & 14 & 3 & 1 & 3 & 2 & 17 \\
\hline Chan et $\mathrm{al}^{19}$ & 53 & - & 4 & 1 & - & - & 1 \\
\hline Faucheron et $\mathrm{al}^{22}$ & 29 & - & 1 & 2 & & 1 & 4 \\
\hline Ganio et $\mathrm{al}^{50}$ & 16 & 15.5 & - & - & - & 1 & 0 \\
\hline Dudding et $\mathrm{al}^{51}$ & 48 & 51 & - & 2 & 1 & - & 1 \\
\hline Gourcerol et al ${ }^{39}$ & 33 & 12 & 4 & - & - & - & - \\
\hline Govaert et al ${ }^{40}$ & 155 & 28.1 & - & 5 & - & - & 14 \\
\hline Hetzer et $\mathrm{a}^{41}$ & 37 & - & - & 2 & 1 & 7 & 7 \\
\hline Holzer et $\mathrm{al}^{42}$ & 7 & 32 & - & 1 & - & 1 & 2 \\
\hline Jarret et $\mathrm{al}^{43}$ & 46 & 12 & 3 & - & 4 & - & 5 \\
\hline Kenefick et $\mathrm{al}^{52}$ & 15 & 24 & - & - & 2 & 4 & 2 \\
\hline Leroi et al ${ }^{44}$ & 6 & 2 & - & - & 1 & 1 & 0 \\
\hline Malouf et a $1^{45}$ & 5 & 16 & - & - & 1 & - & 1 \\
\hline Matzel et al ${ }^{46}$ & 34 & 24 & 10 & 1 & 1 & - & 6 \\
\hline Matzel et $\mathrm{al}^{23}$ & 12 & 9,8 años & 2 & - & - & 2 & 5 \\
\hline Melenhorst et al ${ }^{26}$ & 100 & 25,5 & - & 1 & - & - & 7 \\
\hline Michelsen et al $^{27}$ & 142 & 24 & - & 2 & - & - & 31 \\
\hline Rasmussen et al ${ }^{17}$ & 37 & 6 & - & 2 & - & - & 5 \\
\hline Rosen et $\mathrm{al}^{53}$ & 16 & 15 & - & 3 & 2 & - & 6 \\
\hline Tjandra et $\mathrm{al}^{25}$ & 53 & 12 & - & - & - & - & 0 \\
\hline Uludag et $\mathrm{a}^{49}$ & 62 & 12 & - & 2 & - & - & 12 \\
\hline Wexner et $\mathrm{al}^{16}$ & 120 & 28 & 37 & 5 & 5 & 2 & - \\
\hline
\end{tabular}

La reprogramación del generador y analgésicos es el tratamiento más habitual ${ }^{24}$. Wexner et $\mathrm{al}^{16}$, comunican una tasa de dolor y parestesias en el $25 \%$ de los pacientes, resuelto mediante tratamiento médico, y que no requirieron el explante en ningún caso. Tjandra et $\mathrm{al}^{25}$, describen la aparición de dolor en el $6 \%$ de los pacientes, y molestias vaginales en el 9\%. La implantación del generador en la pared abdominal se ha relacionado con un aumento de la incidencia de este efecto adverso ${ }^{17}$. En nuestra serie hemos observado la presencia de dolor en el lugar del implante, con episodios de dolor agudo y parestesias en miembros inferiores, y que fue refractario a tratamiento médico y requirieron el explante del dispositivo. Aunque la frecuencia de aparición es pequeña (3 pacientes), hemos comprobado que su intensidad ha sido mayor a la comunicada en la literatura.

En un paciente de la serie, decidimos la retirada del implante para la realización de un resonancia magnética. Se ha descrito en la bibliografía roturas del generador durante la realización de una resonancia magnética ${ }^{26}$, por lo que decidimos esta retirada.

Durante el seguimiento, hemos tenido que cambiar el generador en 3 pacientes por presentar agotamiento de la batería. La duración de esta depende del voltaje necesaria durante el tratamiento. Con el fin de aumentar la vida útil del implante, Michelsen et al, propusieron que en ciertos pacientes se desconecte durante la noche ${ }^{27}$.

La rotura o desplazamiento de electrodos o la rotura del generador es muy poco frecuente. Se ha descrito sobre todo tras traumatismos sobre la región glútea. Normalmente requieren la reintervención del paciente y la sustitución de la parte dañada. Es una complicación fácilmente subsanable, pudiendo intervenirse al paciente con anestesia local. En nuestra serie, uno de los pacientes sufrió la rotura del electrodo tras una caída, sustituyéndose el mismo, 
y presentando la misma eficacia previa al traumatismo. En otro paciente, durante un parto vaginal se movilizó el electrodo, y se tuvo que cambiar, presentando los mismo resultados clínicos ${ }^{28}$.

En comparación con otras técnicas quirúrgicas (graciloplastía, esfínter artificial...) la neuroestimulación de raíces sacras presenta un menor número de complicaciones funcionales durante el seguimiento. En ninguno de nuestros pacientes hemos objetivado impactación fecal o estreñimiento tras el tratamiento, complicación muy frecuente en la técnica del esfínter anal artificial. Así mismo, complicaciones tan graves como sepsis, ulceración, migración del dispositivo entre otras, muy frecuentes cuando se implanta un esfínter anal artificial ${ }^{10,11,29-34}$, no se han presentado en ninguno de los pacientes tratados en nuestro centro mediante la estimulación de raíces sacras. Meurette et $\mathrm{al}^{35}$, compararon 15 pacientes tratados satisfactoriamente con estimulación de raíces sacras con 15 pacientes a los que se les implantó un esfínter anal artificial. Si bien aunque los resultados respecto a la escala de Wexner para el cálculo de la incontinencia fecal fueron mejores en el grupo del esfínter anal artificial, con diferencias estadísticamente significativas, el número de complicaciones y episodios de estreñimiento fueron superiores en el segundo grupo, también con diferencias estadísticamente significativas. En cuanto al resultado obtenido respecto a la calidad de vida postoperatoria, no se encontraron diferencias entre uno y otro procedimiento quirúrgico. Wong et $\mathrm{al}^{31}$, en un estudio multicéntrico sobre la implantación de un esfínter artificial para el tratamiento de la incontinencia fecal, describen infección en el 34\% de los pacientes, necesitando revisión quirúrgica en el 75\% de los casos. Los pacientes también experimentaron erosión en el área de implantación, dolor durante la activación, estreñimiento e impactación fecal. La tasa de explante fue superior al 35\%. Complicaciones muy superiores a las comunicadas con la terapia neuroestimuladora.

Matzel et $\mathrm{al}^{13}$, en la serie con más pacientes incluidos, describen tasas de complicación de la graciloplastía dinámica superiores al $75 \%$, consideradas graves en el $50 \%$ de los pacientes, si bien consideran tratables la mayoría de ellas. Baeten et $\mathrm{al}^{36}$, comunican una tasa de efectos adversos similar (74\%). Estamos, por tanto, ante un tratamiento quirúrgico con un índice de complicaciones muy superior a la neuromodulación de raíces sacras.

\section{Conclusiones}

En conclusión, la terapia con neuroestimulación de raíces sacras en el tratamiento de la incontinencia fecal grave es un tratamiento seguro, con un bajo índice de complicaciones, pero como toda técnica quirúrgica debe ser realizada por personal experto en ese campo, para obtener los resultados óptimos.

\section{Referencias}

1.- Baxter NN, Rothenberger DA, Lowry AC. Measuring fecal incontinence. Dis Colon Rectum 2003;46:15911605.

2.- Bharucha AE, Locke GR 3rd, Seide BM, Zinsmeister AR. A new questionnaire for constipation and faecal incontinence. Aliment Pharmacol Ther. 2004;20:355-64.

3.- Eypasch E, Williams JI, Wood Dauphinee S, Ure BM, Schmülling C, Neugebauer et al. Gastrointestinal quality of life index: Development, validation and application of a new instrument. Br J Surg. 1995;82:216-22.

4.- Matzel KE, Stadelmaier U, Hohenfellner M, Gall FP. Electrical stimulation for the treatment of fecal incontinence. Lancet 1995;346:1124-7.

5.- Jorge JMN, Wexner SD. Etiology and management of fecal incontinence. Dis Colon Rectum 1993;36:77-97.

6.- Navarro JM, Arroyo Sebastián A, Pérez Vicente F, Sánchez Romero AM, Pérez Legaz J, Serrano Paz P, et al. Sacral root neuromodulation as treatment for fecal incontinence. Preliminary results. Rev Esp Enferm Dig. 2007;99:636-42.

7.- Nikiteas N, Korsgen S, Kumar D, Keighley MR. Audit of sphincter repair. Factors associated with poor outcome. Dis Colon Rectum 1996;39:1164-70.

8.- Morren GL, Hallbook O, Nystrom PO, Baeten CG, Sjödahl R. Audit of anal sphincter repair. Colorectal Dis. 2001;3:17-22.

9.- Setti Carraro P, Kamm MA, Nicholls RJ. Long-term results of postanal repair for neurogenic faecal incontinence. Br J Surg. 1994; 81:149-54.

10.- Devesa JM, Rey A, Hervas PL, Halawa KS, Larrañaga I, Svidler L, et al. Artificial anal sphincter: complications and functional results of a large personal series. Dis Colon Rectum 2002;45:1154-63.

11.- Christiansen J, Rasmussen OO, Lindorff-Larsen K. Long-term results of artificial anal sphincter implantation for severe anal incontinence. Ann Surg. 1999;230:45-8

12.- Konsten J, Baeten CG, Spaans F, Havenith MG, Soeters PB. Follow-up of anal dynamic graciloplasty for fecal continence. World J Surg. 1993; 17: 404-9.

13.- Matzel KE, Madoff RD, LaFontaine LJ, Baeten CG, Buie WD, Christiansen J, et al. Dynamic Graciloplasty Therapy Study Group. Complications of dynamic graciloplasty: incidence, management, and impact on outcome. Dis Colon Rectum 2001;44:1427-35.

14.- Konsten J, Baeten CG, Spaans F, Havenith MG, Soeters PB. Follow-up of anal dynamic graciloplasty for fecal continence. World J Surg. 1993;17:404-9.

15.- Muñoz A, Navarro A. Estimulación de raíces sacras 
en el tratamiento de la incontinencia fecal. Cir Esp. 2010;87:271-2.

16.- Wexner SD, Coller JA, Devroede G, Hull T, McCullum R, Chan M, et al. Sacral Nerve Stimulation for Fecal Incontinence: Results of a 120-Patient Prospective Multicenter Study. Ann Surg. 2010;251:441-9.

17.- Rasmussen OO, Buntzen S, Sorensen M, Laurberg S, Christiansen J. Sacral nerve stimulation in fecal incontinence. Dis Colon Rectum 2004;47:1158-63.

18.- Jarrett ME, Mowatt G, Glazener CM, Fraser C, Nicholls RJ, Grant AM, et al. Systematic review of sacral nerve stimulation for faecal incontinence and constipation. $\mathrm{Br}$ J Surg. 2004; 91:1559-69.

19.- Chan M, Tjandra J. Sacral Nerve Stimulation for Fecal Incontinence: External Anal Sphincter Defect vs. Intact Anal Sphincter Dis Colon Rectum 2008;51:1015-25.

20.- Melenhorst J, Koch SM, Uludag O, van Gemert WG, Baeten CG. Sacral neuromodulation in patients with faecal incontinence: results of the first 100 permanent implantations. Colorectal Dis. 2007;9:725-30.

21.- Michelsen HB, Thompson-Fawcett M, Lundby L, Krogh K, Laurberg S, Buntzen S. Six Years of Experience With Sacral Nerve Stimulation for Fecal Incontinence. Dis Colon Rectum 2010;53:414-21.

22.- Faucheron JL, Voirin D, Badic B. Sacral Nerve Stimulation for fecal Incontinence: Causes of Surgical Revision From a Series of 87 Consecutives Patients Operated on in a Single Institution. Dis Colon Rectum 2010:53;1501-7.

23.- Matzel KE, Lux P, Heuer S, Besendörfer M, Zhang W. Sacral nerve stimulation for faecal incontinence: longterm outcome. Colorectal Dis. 2009;11:636-41.

24.- Gallas S, Michot F, Faucheron JL, Meurette G, Lehur PA, Barth X, et al. Predictive factors for successful sacral nerve stimulation in the treatment of faecal incontinence: results of trial stimulation in 200 patients. Colorectal Dis. 2011;13:689-96.

25.- Tjandra JJ, Chan MK, Yeh CH, Murray-Green C. Sacral nerve stimulation is more effective than optimal medical therapy for severe fecal incontinence: a randomized, controlled study. Dis Colon Rectum 2008;51:494-502.
26.- Melenhorst J, Koch SM, Uladag O, van Gemert WG, Baeten CG. Is a morphologically intact anal sphincter necessary for success with sacral nerve modulation in patients with faecal incontinence? Colorectal Dis. 2008;10:257-62.

27.- Michelsen HB, Krogh K, Buntzen S, Laurberg S. A prospective randomized study: switch off the sacral nerve stimulator during the night? Dis Colom Rectum 2008; 51: 538-540.

28.- Moya P, Navarro JM, Arroyo A, López A, Ruiz-Tovar J, Calpena R. Sacral nerve stimulation During Pregnancy in patients with severe fecal incontinence. Tech Coloproctol. 2013;17:245-6.

29.- Casal E, Ildefonso S, Carracedo R, Facal C, Sánchez JA. Artificial bowel sphincter in severe anal incontinence. Colorectal Dis. 2004;6:180-4 .

30.- O'Brien PE, Skinner S. Restoring control: the Acticon Neosphincter artificial bowel sphincter in the treatment of anal incontinence. Dis Colon Rectum 2000;43: 12136.

31.- Wong WD, Congliosi SM, Spencer MP, Corman ML, Tan P, Opelka FG, et al. The safety and efficacy of the artificial bowel sphincter for fecal incontinence: results from a multicenter cohort study. Dis Colon Rectum 2002;45:1139-53.

32.- Finlay IG, Richardson W, Hajivassiliou CA. Outcome after implantation of a novel prosthetic anal sphincter in humans. Br J Surg. 2004;91:1485-92.

33.- Ortiz H, Armendariz P, DeMiguel M, Ruiz MD, Alós $\mathrm{R}$, Roig JV. Complications and functional outcome following artificial anal sphincter implantation. Br J Surg. 2002; 89:877-81.

34.- Altomare DF, Binda GA, Dodi G, La Torre F, Romano $\mathrm{G}$, Rinaldi M, et al. Disappointing long-term results of the artificial and sphincter for faecal incontinence. Br J Surg. 2004;91:1352-3.

35.- Meurette G, La Torre M, Regenet N, Robert-Yap J, Lehur PA. Value of sacral nerve stimulation in the treatment of severe faecal incontinence: a comparison to the artificial bowel sphincter. Colorectal Dis. 2009; 11:631-5. 